\title{
Independent saturation of three TrpRS subsites generates a partially assembled state similar to those observed in molecular simulations
}

\author{
Poramaet Laowanapiban ${ }^{a, 1}$, Maryna Kapustinab ${ }^{b}$ Clemens Vonrheinc, Marc Delarue ${ }^{\mathrm{d}}$, Patrice Koehle, \\ Charles W. Carter, Jr. ${ }^{\mathrm{a}, 2}$ \\ aDepartment of Biochemistry and Biophysics, University of North Carolina, Chapel Hill, NC 27599; bepartment of Cell Biology, University of North Carolina, \\ Chapel Hill, NC 27599; 'Globalphasing Ltd., Cambridge CB3 OAX, United Kingdom; dDynamique Structurale des Macromolecules, Unité de Recherche \\ Associée 2185 du Centre National de la Recherche Scientifique, Pasteur Institute, 75015 Paris, France; and eGenome Center and Department \\ of Computer Sciences, University of California, Davis, CA 95616
}

Communicated by Paul Schimmel, The Scripps Research Institute, La Jolla, CA, December 15, 2008 (received for review October 12, 2008)

Two new crystal structures of Bacillus stearothermophilus tryptophanyl-tRNA synthetase (TrpRS) afford evidence that a closed interdomain hinge angle requires a covalent bond between AMP and an occupant of either pyrophosphate or tryptophan subsite. They also are within experimental error of a cluster of structures observed in a nonequilibrium molecular dynamics simulation showing partial active-site assembly. Further, the highest energy structure in a minimum action pathway computed by using elastic network models for Open and Pretransition state (PreTS) conformations for the fully liganded TrpRS monomer is intermediate between that simulated structure and a partially disassembled structure from a nonequilibrium molecular dynamics trajectory for the unliganded PreTS. These mutual consistencies provide unexpected validation of inferences drawn from molecular simulations.

conformational transition state | domain movement | induced fit | minimum action pathway | nonequilibrium molecular dynamics

Crystal structures of enzymes provide a limited subset of their possible biochemical states and cannot provide details of catalytic conformational changes. Coordinated changes that align catalytic residues properly when substrates or analogs bind are referred to as "induced-fit." By definition, such changes involve transitions to conformations that are not favored without ligands. It follows that they "store" conformational free energy. Dynamic interactions along pathways between stable crystal structures are therefore of paramount interest if we are to understand molecular details of conformational free-energy transduction.

Linking crystal structures together to form a mechanistic pathway requires procedures that transcend experimental structure determination $(1,2)$. Molecular simulation techniques to characterize such pathways range from hybrid quantum mechanical/ molecular mechanics (QM/MM) (3) and molecular dynamics (MD) (4) to discrete molecular dynamics (DMD) $(5,6)$ and minimumaction pathway (MAP) (7) computations. Validating these methods relies mostly on comparison with modeling done for known structural states. Only rarely does a new structure compare favorably with predictions based on previous simulations. Such an example is provided by crystal structures of Bacillus stearothermophilus tryptophanyl-tRNA synthetase (TrpRS) complexed with fragments bound to its adenosine, tryptophan, and pyrophosphate subsites, solved to test a hypothesis about the structural requirements for induced fit.

Acylation of tRNA ${ }^{\text {Trp }}$ proceeds by 2 half-reactions, tryptophan activation by ATP and acyl-transfer to cognate tRNA. Amino acid activation proceeds quite slowly in water (8), whereas mixed anhydrides react rapidly with water and alcohols (9). Thus, the most important catalytic rate acceleration possessed by aminoacyl-tRNA synthetases, by many orders of magnitude, is acyl-group activation by ATP. The structural reaction profile for tryptophan activation by TrpRS has been elucidated by structural, biochemical, and bio- physical studies. Four structural subclasses have been identified with catalysis of Trp-5'-AMP formation (10-15). Relationships between structures and their catalytic relevance have been extensively documented $(13,15)$, in particular, by how they rationalize the kinetic behavior of active-site mutations in the closely related tyrosyl-tRNA synthetase $(16,17)$.

Two TrpRS structures, an Open conformation (12) and a closed, pretransition state (PreTS) conformation (13), represent initial and final states of a substantial conformational change that activates $\mathrm{Mg}^{2+} \cdot$ ATP before its reaction with tryptophan (13). Conformational free energy is stored during this induced fit (15) and recovered in a subsequent catalytic step (11). Hinge bending and twisting domain movements associated with these conformational changes are correlated with ligands bound to the Open, closed PreTS (13), and Products (11) states (Fig. 1).

The closed-hinge angle appears to depend solely on binding either ATP or Trp-5'-AMP, to both subunits of the dimer, whereas the twist requires $\mathrm{Mg}^{2+} \cdot \mathrm{ATP}$ (18). Both ligands possess a highenergy bond between AMP and either $\mathrm{PP}_{\mathrm{i}}$ in the closed PreTS or tryptophan in the closed, Products state, respectively. They induce hinge closure irrespective of whether the third subsite is occupied (Fig. 1) (19). Thus, the covalent linkage seems critical for inducing and/or stabilizing domain closure.

We were interested in whether saturating the active subsites with tryptophan, adenosine, and $\mathrm{PP}_{\mathrm{i}}$ fragments would induce a closed conformation. We therefore prepared and solved X-ray structures in which the enzyme was provided with saturating concentrations of these 3 ligands. Posing this experimental question is a structural counterpart to Wolfenden's studies of fragment binding thermodynamics $(20,21)$.

The new structures extend understanding of TrpRS catalysis three distinct ways. First, they support the hypothesis that a covalently linked adenosine moiety is necessary to stabilize closed TrpRS conformations. Second, they document the high affinity of the KMSKS loop for the $\beta$-phosphate, reinforcing earlier suggestions $(13,15)$ that this affinity helps develop additional binding energy in a dissociative transition state. Finally, they provide unexpected experimental support and rationalization for nonequi-

Author contributions: P.L. and C.W.C. designed research; P.L., M.K., C.V., and M.D. performed research; C.V., M.D., and P.K. contributed new reagents/analytic tools; P.L., M.K., C.V., M.D., and C.W.C. analyzed data; and P.L. and C.W.C. wrote the paper.

The authors declare no conflict of interest.

Data deposition: The atomic coordinates and structure factors have been deposited in the Protein Data Bank, www.pdb.org (PDB ID codes 3FHJ and 3FI0).

1Present address: Department of Microbiology (Faculty of Science) Mahidol University, Bangkok 10400, Thailand.

${ }^{2}$ To whom correspondence should be addressed. E-mail: carter@med.unc.edu.

This article contains supporting information online at www.pnas.org/cgi/content/full/ 0812752106/DCSupplemental.

() 2009 by The National Academy of Sciences of the USA 

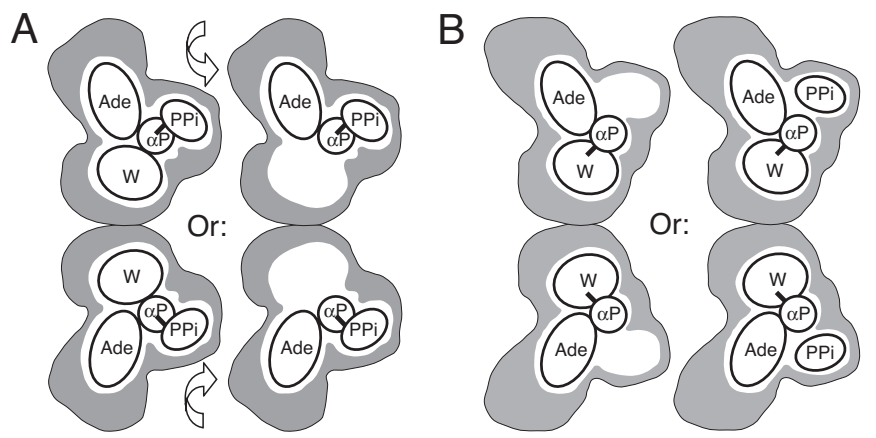

C

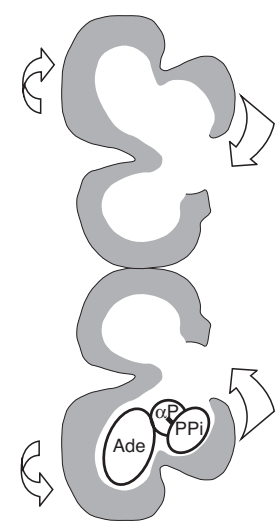

D

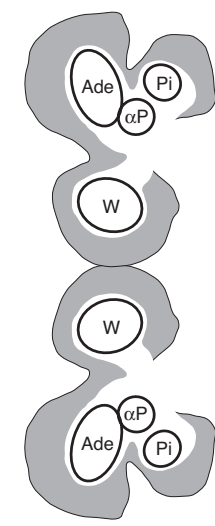

Fig. 1. Ligand dependence of TrpRS conformations. (A) Pre-TS structures formed with $\mathrm{Mg}^{2+}$.ATP and tryptophanamide or with $>10 \mathrm{mM} \mathrm{Mg}^{2+} \cdot$ ATP. (B) Product conformations formed by in situ synthesis of trp-5'-AMP or by cocrystallization with stable adenylate analogs. (C) Open structures, shown here with substoichiometric $\mathrm{Mg}^{2+} \cdot$ ATP. Wide and narrow arrows denote hinge bending and twisting, respectively, converting $C$ to $A$ to $B$. (D) WMP structures solved in this work.

librium simulations suggesting a conformational free-energy barrier early in the induced-fit phase of the reaction.

\section{Results}

We use the acronym WMP for the new structures, from the 3 defining ligands, tryptophan, AMP, and phosphate. We discuss, in turn, the ligands, the overall conformations compared with known structures, and finally, relationships to 3 nonequilibrium computational simulations relevant to understanding the induced-fit conformational energetics.

Previous Open TrpRS structures were solved by molecular replacement, which proved unusually difficult (12). Accordingly, 3-wavelength multiple anomalous dispersion (MAD) and native datasets were recorded for experimental phase determination. The SeMet-derivatized TrpRS complex was solved at 2.7-Å resolution in space group P1 with selenium anomalous scattering-derived experimental phases, molecular replacement, and density modification. The native TrpRS complex was solved at 2.5-Å resolution from that structure by molecular replacement in space group C2.

Whereas monoclinic native WMP complex crystals have the expected 3 dimers per asymmetric unit, the selenomethioninesubstituted triclinic cell has 9 dimers. Electron density maps with either experimental or later refined phases all persistently showed considerable variability for the AMP. Efforts to impose a highersymmetry space group were unsuccessful. Imposing an idiosyncratic variety of noncrystallographic symmetries improved phases substantially. We believe, therefore, that the structures represent an ensemble of different microstates.

The WMP Ligands. The AMP and tryptophan ligands occupied the expected binding subsites. Consistent with the relative domain
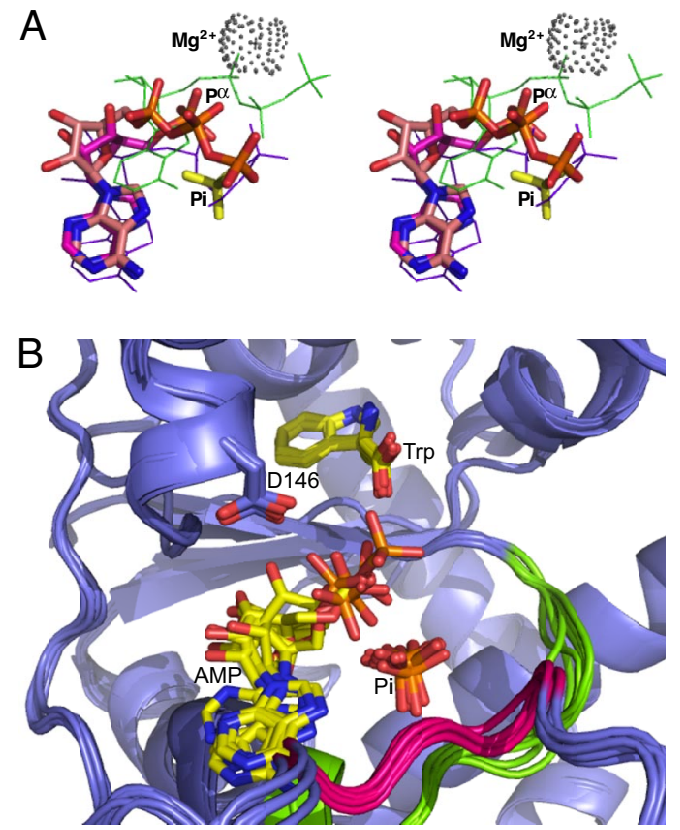

Fig. 2. Adenosine positions in the TrpRS WMP complexes. Monomers were superimposed by using 42 residues derived from the most constant portions of the Rossmann fold (10). (A) Comparison between the representative SeMet WMP structure, the simulated structure (magenta and wheat sticks, respectively) and the Open [1MAW(A)] and PreTS (1MAU) structures (blue and green lines, respectively). (B) Monomers a-f of the SeMet-substituted complex. The HIGH catalytic signature is green; the KMSKS signature is magenta. Tryptophan and $\mathrm{P}_{\mathrm{i}}$ moieties superimpose closely; AMP assumes many different positions close to that observed in the singly liganded ATP complex (13). The active site aspartate, D146, interacts with the ribose $2^{\prime}-\mathrm{OH}$ group in the assembled PreTS active site, but is too far from the ribose in the WMP complexes to stabilize a unique structure.

orientations described below, the adenosine moiety assumes a position intermediate between those in the Open and PreTS crystal structures (Fig. 2A) and nearly coincident with that observed in a partially closed structure seen previously in MD simulations (18). However, crystallization requires $2.0 \mathrm{M}$ phosphate and, at this concentration, inorganic phosphate, rather than the anticipated $\mathrm{PP}_{\mathrm{i}}$ group, bound to the $\mathrm{PP}_{\mathrm{i}}$ binding sites. This moiety nonetheless invariably assumed the position of the $\beta$-phosphate of the $\mathrm{PP}_{\mathrm{i}}$ leaving group, which, because of the low twist angle, is $4.43 \pm 0.33 \AA$ from the $\alpha$-phosphate compared with $2.98 \AA$ in ATP, a point to which we return in the discussion.

The intact adenosine binding site in the PreTS combines interactions with both domains, whereas the tryptophan subsite is relatively unaffected by domain motion. Although tryptophan and $P_{i}$ positions are quite reproducible in all 24 monomers, AMP moieties are much more variable (Fig. 2B). Mean excursions of $P_{i}$ and tryptophan moieties in superimposed structures (Table 1) are 5 and 4 times smaller, respectively, than those of the AMP moiety. Moreover, experimentally phased electron density maps show 2 of the 24 different bound AMP molecules binding in different orientations; we believe they are legitimate nonproductive binding modes. A key indicator of adenosine site fragmentation, the ribose

Table 1. Reproducibility of ligand positions in SeWMP monomers A-F

\begin{tabular}{lccc} 
Distance & ${ }^{\alpha} \mathrm{P}-\alpha \mathrm{P}(\mathrm{AMP}), \AA$ & $\mathrm{C}-\mathrm{C}(\operatorname{Trp}), \AA$ & $\mathrm{P}_{\mathrm{i}}-\mathrm{P}_{\mathrm{i}}, \AA$ \\
\hline Mean, $\AA$ & 1.38 & 0.34 & 0.27 \\
Standard deviation, $\AA$ & 0.90 & 0.12 & 0.13 \\
\hline
\end{tabular}




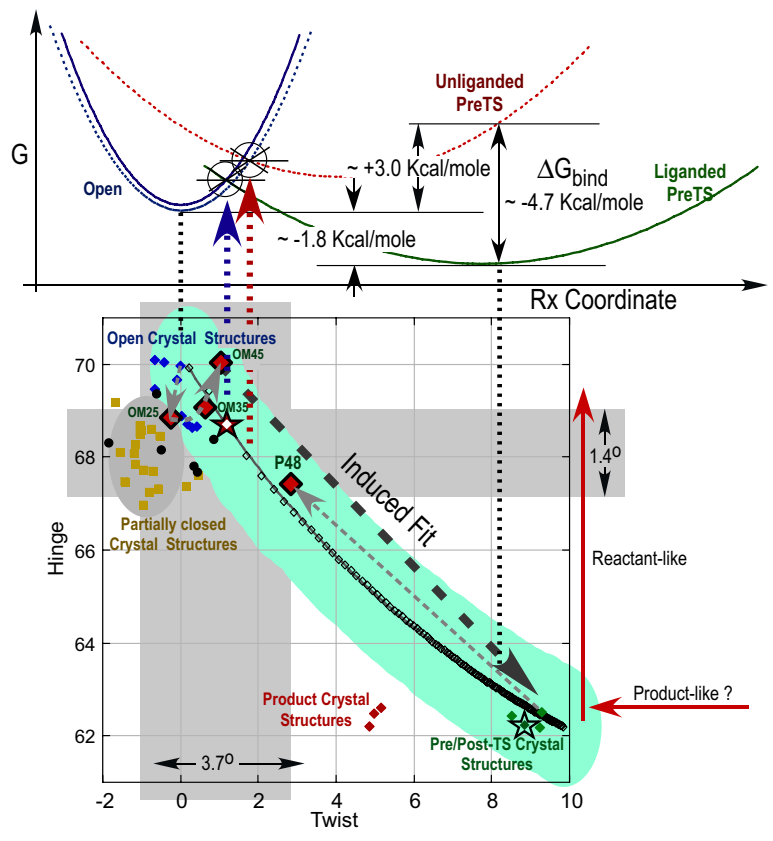

Fig. 3. Conformational space defined by internal conformational angles of TrpRS crystal structures and simulations. Open, PreTS, and Products complex crystal structures are indicated by blue, green, and red diamonds (7). Because the 3 Open state crystal structures are isomorphous, there are $<18$ distinct blue squares. Partially closed native and SeMet WMP structures are shown as black circles and amber squares. MD structures (large, filled red squares) and the origins of their trajectories (fine gray dashed arrows; OM25 and P48) are described in the text. Open squares denote structures along a minimum action pathway connecting the Open and PreTS structures. The red filled star represents the highest-energy structure along the simulated path. Dashed (unliganded) and solid (bound to $\mathrm{Mg}^{2+}$.ATP and tryptophan) semiempirical harmonic conformational energy wells were inferred from experimental conformational free-energy changes with and without ATP (15) and from simulations. Circles denote transition states along the forward and reverse induced-fit transition. Vertical dashed lines relate free-energy minima (gray; observed crystal structures) and maxima (simulations; red, blue arrows) to structures below.

$2^{\prime}-\mathrm{OH}$ (Fig. 2), is $6.22 \pm 0.51 \AA$ from the D146 carboxylate, compared with $7.18 \pm 0.74 \AA$ in the Open ATP complex, 1MAW, consistent with partial closing of the hinge. In the PreTS state, this distance is $2.64 \AA$ denoting a hydrogen bond.

Conformational Effects of Substrate Fragments and Selenomethionine Substitution. We relate WMP structures to the structural reaction profile by projecting structural differences to internal coordinates defined by 2 interdomain angles (18) (supporting information (SI) Text, Internal Angles Defining Domain Orientation; Fig. S1). As was true of their closest structural relatives, the previously solved Open states [1D2R (12), 1MB2, and 1MAW (13)], variation in crystallographically independent molecules reduces the variance of the mean domain orientations of the WMP complexes. This statistical precision allowed us to identify 2 significant structural differences by regressing the internal coordinates against chemical parameters (SI Text, Regression Analyses of Domain Positions Against Ligands and Selenomethionine Substitution; Tables S1 and S2). WMP complexes are more closed than other Open structures, and selenomethionine complexes are untwisted, relative to native WMP complexes

Two principal components derived from Tetrada structural scoring (22) span $84 \%$ of the variance in all TrpRS crystal structures solved to date. These 2 principal components, PC1 and PC2, are very nearly equivalent $\left(R^{2}=0.99\right)$ to the hinge and twist angles (in degrees) by transformations in Eq. 1:
Table 2. Mean TrpRS conformational angles

\begin{tabular}{|c|c|c|c|c|}
\hline Structural cluster & $\langle$ Hinge $\rangle$ & $\sigma($ Hinge $)$ & $\langle$ Twist $\rangle$ & $\sigma$ (Twist \\
\hline \multicolumn{5}{|l|}{ Crystal structures (number) } \\
\hline Open, unliganded (6)* & 69.3 & 0.98 & -0.51 & 0.43 \\
\hline Open, singly liganded $(12)^{\dagger}$ & 69.2 & 0.61 & -0.07 & 0.33 \\
\hline WMP (6) 3FHJ & 68.3 & 0.6 & -0.2 & 1.0 \\
\hline SeWMP (18) 3FI0 & 68.0 & 0.6 & -0.9 & 0.5 \\
\hline PreTS (5) 1MAU, $1 \mathrm{M} 83$ & 62.5 & 0.24 & 8.9 & 0.34 \\
\hline $\begin{array}{l}\text { Products } 116 \mathrm{~K}, 116 \mathrm{~L}, 1 \mathrm{i} 6 \mathrm{M} \\
\text { Simulated structures }\end{array}$ & 62.3 & 0.23 & 4.6 & 0.12 \\
\hline $\begin{array}{l}\text { OM25; partially assembled, } \\
2.5 \mathrm{~ns}\end{array}$ & 68.9 & $0.57^{\ddagger}$ & -0.36 & $0.60^{\ddagger}$ \\
\hline $\begin{array}{l}\text { OM45, partially assembled, } \\
4.5 \text { ns }\end{array}$ & 70.25 & $0.57^{\ddagger}$ & 1.31 & $0.60^{\ddagger}$ \\
\hline MinActPath transition state & 67.8 & NA & 1.74 & NA \\
\hline P48; partially disassembled & 67.5 & $0.63^{\ddagger}$ & 2.61 & $1.07^{\ddagger}$ \\
\hline
\end{tabular}

Numbers in parentheses denote the number of crystallographically independent structures.

*The 6 monomer structures from PDB ID code 1D2R.

${ }^{\dagger}$ Six independent monomers each from PDB ID codes $1 \mathrm{MAW}$ and $1 \mathrm{MB} 2$.

₹Standard deviations obtained from variances of the mean as described in SI Text, Statistical Error in Molecular Simulations.

$$
\begin{gathered}
\text { (hinge twist) }=\left\lfloor\begin{array}{cc}
-1.63 & -0.49 \\
1.32 & 1.76
\end{array}\right\rfloor \cdot\left\lfloor\begin{array}{l}
\mathrm{PC} 1 \\
\mathrm{PC} 2
\end{array}\right\rfloor+\left\lfloor\begin{array}{c}
65.73 \\
3.44
\end{array}\right\rfloor \\
{[\mathrm{PC} 1 \mathrm{PC} 2]=\left\lfloor\begin{array}{cc}
-0.785 & -0.216 \\
0.720 & 0.580
\end{array}\right\rfloor \cdot\left\lfloor\begin{array}{c}
\text { hinge } \\
\text { twist }
\end{array}\right\rfloor+\left\lfloor\begin{array}{c}
52.3 \\
-40.60
\end{array}\right\rfloor}
\end{gathered}
$$

Thus, the hinge $(\alpha)$ and twist $(\gamma)(14,18)$ provide a more sensitive and less ambiguous description of domain movement along the structural reaction coordinate than rmsd values. The conformational cycle constitutes a scalene triangle in this plane. Open structures occupy the upper left, open-hinge, untwisted corner; PreTS structures occupy the lower right, closed, twisted corner; and Products structures constitute an intermediately twisted, closed state (Fig. 3).

WMP complexes differ in detail from Open state structures (Table 2). The W, M, and P ligand fragments favor a partially closed hinge. Approximately $45 \%$ of the variance in the hinge angle can be attributed to the WMP ligands, with $P(t)<0.0001$ for the mean difference of $1.14^{\circ}$ between all Open and all WMP states (SI Text, Regression Analyses of Domain Positions Against Ligands and Selenomethionine Substitution). This hinge angle closing is $\approx 15 \%$ of the total difference between the Open and Closed states. Further, the WMP domain orientations are untwisted relative to the OPEN structures. Thus, the substrate fragments partially close the hinge, but, without a covalent bond to pull the subsites together, these ligands cannot convert any of their binding energies into the $\approx 3$ $\mathrm{kcal} / \mathrm{mol}$ of conformational free energy required to close and twist the domains, creating the PreTS (13) structure.

The average hinge angles $\left(68.3^{\circ}, 68.0^{\circ}\right)$ of the native and SeMet substituted structures are not significantly different. However, the mean twist angle for SeMet-containing WMP complexes $(-0.88)$ is $\approx 0.6^{\circ}$ smaller than those of the native structures $\left(-0.23^{\circ}\right)$. When account is taken of the covariation of hinge and twist among different structures, regression analysis shows that the twist angle is negatively correlated with the presence of selenomethionine (SI Text, Regression Analyses of Domain Positions Against Ligands and Selenomethionine Substitution; $P(t)=0.02)$. SeMet substitution is therefore a likely cause of the different WMP and SeWMP crystal habits. 

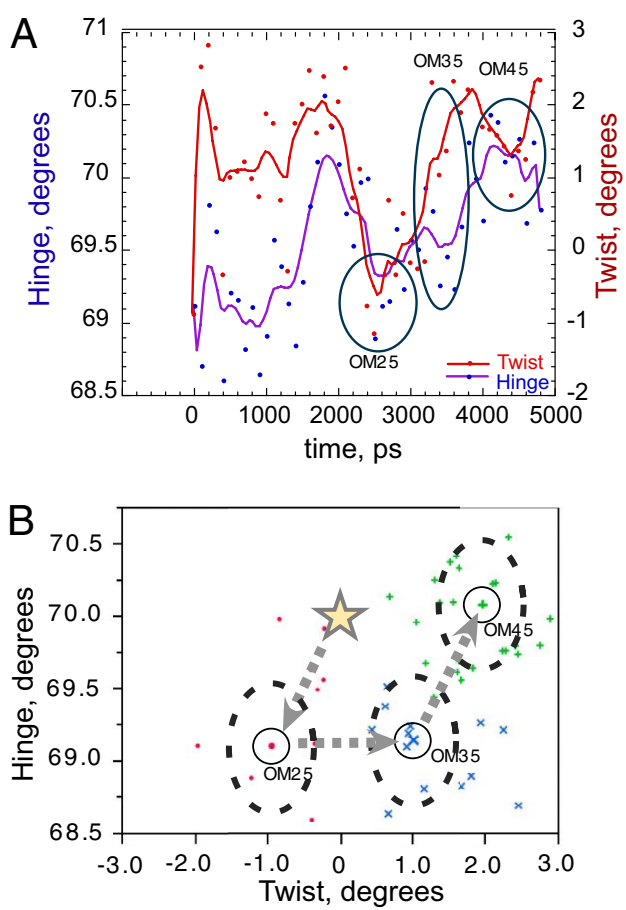

Fig. 4. The OM partial assembly trajectory. $(A)$ Time courses of the 2 internal coordinates. The star denotes the initial configuration before energy minimization, which carries the system immediately to a configuration that recurs later in the trajectory (OM25). (B) Three structural clusters based on distances derived from the first 2 principal components (which map to the hinge and twist angles), represented by snapshots at 2,500 (OM25, red), 3,500 (OM35, blue), and 4,500 (OM45, green) ps. Radii of dashed ellipses are $\sigma$ values from Table 2.

The WMP Structures Help Rationalize Several Nonequilibrium Simulations Related to Induced Fit. Kapustina (18) described both liganded and unliganded MD trajectories computed for all known TrpRS states. Two types of trajectories migrated from the region of the original crystal structure toward another state. A partially assembled complex constructed by adding tryptophan and $\mathrm{Mg}^{2+} \cdot \mathrm{ATP}$ to the Open state structure 1MAW progressed toward the PreTS state. A partially disassembled complex constructed by removing $\mathrm{Mg}^{2+} \cdot$ ATP from the PreTS crystal structure 1MAU regressed back almost to the Open conformation. We recently described a possible significance of the latter behavior, noting that ligand-free closed, twisted states derived from $\mathrm{Mg}^{2+}$.ATP (1MAU) and adenosine$3^{\prime}$-tetraphosphate (AQP; 2OV4) complexes evolved in opposite directions along the structural reaction profile, implying the existence of a conformational transition state between the PreTS and PostTS crystal structures (14).

We previously considered the partially assembled (OM45) and partially disassembled (P48) structures to be similar, because they have similar hinge angles. The new WMP structures, however, have hinge angles intermediate between those of the 2 terminal structures. All 3 sets of structures have quite distinct twist angles. We therefore examined how these nonequilibrium simulations compare with crystal structures in light of quantitative error estimates for the simulations (SI Text, Statistical Error in Molecular Simulations) (23). Surprisingly, these are comparable to experimental error estimates (Table 2).

Partial assembly with $\mathrm{Mg}^{2+}$.ATP and tryptophan is both modest and stochastic, oscillating several times between partially closed and partially twisted configurations (Fig. 4A). Using 2 principal components together and error estimates, we identified 3 clusters that occur in approximately equal amounts and approximately in succession (Fig. 4B). The first cluster forms rapidly during the equil- ibration period, and recurs later as representative OM25. Conformational angles of the 6 native WMP structures are, on average, within one standard deviation of those observed for that structure.

The P48 trajectory regresses from the PreTS state toward the Open state when $\mathrm{Mg}^{2+} \cdot$ ATP is removed, opening and untwisting rapidly and monotonically (within the first nanosecond of simulation, with little subsequent change in conformation during 4 additional nanoseconds of simulation (18). Although its final hinge angle is similar to those of the WMP complexes and the OM trajectory, its twist angle, $2.61^{\circ}$, differs by $6 \sigma$, or $\approx 35 \%$ of the full range of the small domain twisting motion. Thus, although chemically identical, the twist angles of the partially assembled and disassembled unliganded TrpRS structures differ sufficiently that they represent distinct states that do not merge from different starting points within 5-ns MD simulations.

Possible resolution of this puzzle (Fig. 3) emerged from a Minimum Action Pathway (MAP) constructed from elastic network models for the fully liganded Open and PreTS conformations to represent the entire forward induced-fit conformational change. The MAP algorithm assumes that the initial and final conformations diffuse within local harmonic potentials (solid parabolas, Fig. 3 Upper), centered on the crystallographic coordinates (thin dashed vertical lines). It differs from MD simulations in that it solves analytical expressions for the structure and energy of the conformational transition state where the 2 potentials intersect (7) at the expense of using more approximate potentials.

The relative free energies of free and bound states in both conformations were determined by experiment (15), leaving only the unknown force constants for the Open and PreTS potentials to be adjusted for optimal alignment with structures Fig. 3 Lower. Moreover, because crystal structures of unliganded TrpRS and its Open complexes with tryptophan and (substoichiometric) ATP are indistinguishable, binding of these ligands separately induces only minimal changes in the potential energy well of Open unliganded TrpRS. However, together, they favor induced fit by stabilizing the PreTS conformation.

The highest energy structure from the MAP pathway, denoted by the star in Fig. 3, occurs early in the conformational change (blue dashed arrow). Moreover, it is intermediate between the $2 \mathrm{MD}$ structures (Table 2). Consequently, it presents an early kinetic barrier to the fully liganded Open, and a late barrier to opening in the P48 MD simulations. The MD trajectories appear to stall on either side of the high-energy conformation between them, the partially assembled trajectory at a twist angle smaller, and the partially disassembled structure at a twist angle greater, than those of the (similar) transition states.

Dashed parabolas in Fig. 3 represent harmonic approximations for unliganded TrpRS. The remarkably rapid equilibration of the P48 trajectory argues that unliganded TrpRS is less stable in the PreTS state than in the Open conformation and that its minimum energy lies close to where that trajectory stalls during the last $4 \mathrm{~ns}$. Inferred potentials for unliganded TrpRS therefore suggest a transition state for the reversal of induced fit close to that for the forward reaction with liganded TrpRS (circles in Fig. 3).

The MAP conformational transition state structure should not be confused with the conformational transition state described recently in ref. 14 between the PreTS $\mathrm{Mg}^{2+} \cdot$ ATP complex and the (posttransition state) complex with adenosine $5^{\prime}$-tetraphosphate, AQP shown in Fig. 3 Lower Right.

\section{Discussion}

Domain motion during induced fit closes and twists the 2 TrpRS domains. The anticodon-binding domain moves as a rigid body with both HIGH and KMSKS catalytic signatures to deliver ATP to the tryptophan carboxyl group in the Rossmann fold (RF). Crystal structures of the WMP complexes with 3 subsite-specific fragments show that TrpRS retains an open conformation when bound simultaneously to AMP, tryptophan, and inorganic phosphate. 
Table 3. Crystallographic data

\begin{tabular}{|c|c|c|c|c|}
\hline Crystal type & Native $\lambda_{0}=1.0 \AA$ & Se derivative $\lambda_{0}=0.97934 \AA$ & Se derivative $\lambda_{0}=0.97945 \AA$ & Se derivative $\lambda_{0}=0.97177 \AA$ \\
\hline Space group & $\mathrm{C} 2$ & P1 & P1 & P1 \\
\hline \multirow[t]{4}{*}{ Unit-cell parameters } & $a=223.60 \AA$ & $a=122.03 \AA$ & $a=122.41 \AA$ & $a=122.31 \AA$ \\
\hline & $b=91.98 \AA$ & $b=122.40 \AA$ & $b=122.74 \AA$ & $b=122.68 \AA$ \\
\hline & & $\alpha=79.91^{\circ} \beta=80.52^{\circ}$ & $\beta=80.59^{\circ} \gamma=79.87^{\circ}$ & $\beta=80.60^{\circ} \gamma=79.88^{\circ}$ \\
\hline & & $\gamma=79.81^{\circ}$ & & \\
\hline Observations & 549,810 & 811,830 & 709,775 & 663,814 \\
\hline$\%$ Completeness & $97.6(77.5)$ & $97.7(97.2)$ & $97.8(97.2)$ & $97.9(97.4)$ \\
\hline$\langle I / \sigma(I)\rangle$ & $19.4(1.68)$ & $11.0(2.4)$ & $10.7(2.3)$ & $11.0(2.8)$ \\
\hline Reflections with $I>3 \sigma(I), \%$ & 65.7 & 87.3 & 85.9 & 82.1 \\
\hline Overall $R_{\text {merge }}$ & 0.081 & 0.069 & 0.079 & 0.074 \\
\hline Redundancy & 3.48 & 3.96 & 3.98 & 3.99 \\
\hline Mosaicity, degrees & 0.25 & 0.54 & 0.54 & 0.54 \\
\hline
\end{tabular}

Values in parentheses represent the highest resolution shell.

Interconverting Binding and Conformational Free Energy. Lacking covalent links between AMP and either $\mathrm{P}_{\mathrm{i}}$ or Trp, the bound WMP ligands do not represent any intermediate state in the tryptophan activation reaction. Bound nucleotide and amino acid subsites in both monomers in WMP dimers and phosphate bound to the KMSKS loop induce only minimal domain closure (Fig. $1 D$ ). It is therefore likely that a covalent bond between the nucleotide and the occupant of another subsite is necessary to induce the closed structures. The respective binding subsites are far enough apart that without a covalent bond to pull sites together, there is no need for TrpRS to overcome the unfavorable free energy $(+3.0 \mathrm{kcal} / \mathrm{mol})$ (15) associated with ligand-free induced fit to retain optimal binding interactions to the separate fragments.

We failed to capture the structure that would establish this point more conclusively, because crystal growth requires $2.0 \mathrm{M}$ phosphate, which competes effectively for $\mathrm{PP}_{\mathrm{i}}$. Stabilization of pregrown crystals in $\left(\mathrm{NH}_{4}\right)_{2} \mathrm{SO}_{4}$, which might allow soaking of $\mathrm{PP}_{\mathrm{i}}$ into preformed crystals, would convolute the question of conformational stability with the unknown influence of crystal packing forces. It therefore may be difficult to resolve this issue fully.

WMP Complex Is Functionally Relevant. The adenosine moiety binds preferentially to the HIGH sequence even when it cannot use the binding of the triphosphate moiety to the neighboring KMSKS loop (13). Two interactions - between D146 and the ribose and between K111 and the $\gamma$-phosphate across the domain boundary-appear to drive induced-fit active-site assembly. The K111 $\varepsilon$-amino group is 13.5-19 $\AA$ from the bound phosphate in the WMP structures and therefore unable to interact. The D146 carboxylate is reproducibly somewhat closer $(6.22 \AA$ vs. $7.18 \AA)$ to the adenosine ribose $2^{\prime}-\mathrm{OH}$ in the WMP structures than it is in the Open ATP complex. Adenosine, by itself, does not induce a closed hinge to create that contact.

The distance between the $\alpha$-phosphate position and the $\mathrm{P}_{\mathrm{i}}$ moiety bound to the KMSKS loop is consistent with that expected for a dissociative transition state for phosphoryl transfer. The low variance of the $\mathrm{P}_{\mathrm{i}}$ position, its coincidence with $\beta$-phosphate in crystals containing a $\mathrm{PP}_{\mathrm{i}}$ leaving group (1MAU, $1 \mathrm{M} 83,2 \mathrm{OV} 4)$, and the high variance of the $\alpha$-phosphate position are likewise consistent with a mechanism that stabilizes the $P_{i}$ leaving group while allowing the $\alpha$-phosphate to migrate toward the tryptophan carboxylate nucleophile.

Corroborating Molecular Simulations. The partially closed WMP structures are similar to structures observed previously only during
MD simulations of fully liganded, Open TrpRS. The relationship of that and the partially opened PreTS simulated structure to the induced-fit pathway was clarified by the early conformational transition state in the MAP pathway based on analytical solution of the structure and energy of the crossing point between elastic network models for the fully liganded Open and PreTS states. These observations afford semiquantitative agreement between the WMP structures, several types of molecular simulations, and experimental free-energy changes for the overall conformational changes and ATP binding (Fig. 3).

Experimental relative free energies of the 4 different structures (15), together with the evidence in all 3 simulations for a barrier with a relatively open hinge and small twist angles, afford a semiempirical calibration of the force constants for the different potentials. In particular, ATP binding appears from Fig. 3 to broaden the PreTS harmonic well, relative to that of the Open state. However, the large numbers of monomers in Open state crystal asymmetric units reflect a range of domain motion in Open TrpRS states, whereas closed-state dimers crystallize with monomers in their crystallographic asymmetric units and diffract to higher resolution $(10,11,13,15)$. These results suggest the opposite conclusion that Open states have relaxed, and closed states have tighter force constants. The puzzling inconsistency between the semiempirical energy surface described in Fig. 3 and that inferred from crystallogenesis suggests that we can further validate or reject the 3 interrelated simulations by comparing the MAP force constants with those obtained by using potentials of mean force (18).

In related work (V. Weinreb, unpublished data), we have used multimutant thermodynamic cycles to confirm active-site lysinemetal interactions whose relative strengths were also first predicted by nonequilibrium MD trajectories (18). These confirmatory results therefore provide important justification for using relatively short, nonequilibrium MD simulations as a way to investigate dynamic aspects of ligand-dependent conformational changes in proteins.

\section{Experimental}

Crystals and Diffraction Data. Native and SeMet TrpRS from B. stearothermophilus were expressed in $E$. coli BL-21 and purified as described in ref. 13. $B$. stearothermophilus crystals were grown by microdialysis in the presence of tryptophan (0.5 mM), AMP (0.01 M), PP $(0.01 \mathrm{M})$, and magnesium $(0.01 \mathrm{M})$. Response-surface analysis (24) revealed a crystal growth optimum in $\approx 2.28 \mathrm{M}$ potassium phosphate at $\mathrm{pH} 6.58$ with $0.6 \% \mathrm{vol} / \mathrm{vol} \mathrm{PEG} 400$ as an additive at $315 \mathrm{~K}$.

Unit-cell parameters for a cryoprotected crystal were $a=122.45 \AA, b=$ $122.86 \AA, c=122.81 \AA, \alpha=79.91^{\circ}, \beta=80.62^{\circ}$, and $\gamma=79.87^{\circ}$. Strong suggestions of higher hexagonal (R3) or possibly monoclinic (C2) space group symmetry were 
ruled out decisively on the basis of unacceptably high $\chi^{2}$ values and merging $R$ factors and subsequently by self-rotation function and native Patterson analysis, which provided unambiguous evidence for the noncrystallographic rotation and translation symmetries.

Native complex crystals had a similar habit, but diffracted to higher resolution and indexed in the higher symmetry space group, $C 2$, with $a=223.60 \AA$, $b=91.98 \AA, c=158.32 \AA, \beta=134.01^{\circ}$ similar to those of monoclinic crystals of other open TrpRS complexes (1MB2 and $1 \mathrm{MAW} ; a=228.73 \pm 0.13 \AA$, $b=$ $\left.92.24 \pm 0.18 \AA, c=157.59 \pm 0.49 \AA, \beta=132.53 \pm 0.26^{\circ}\right)$. Attempts to improve diffraction limits for both crystals by soaking in $3.5 \mathrm{M}$ ammonium sulfate (AS) to dehydrate and anneal crystal packing before cryoprotection in 15\% (vol/ vol) glycerol, $2.5 \mathrm{M}$ AS did not succeed. Flash cooling in $15 \%$ glycerol was equally successful with either crystal form.

SeMet data at 3 wavelengths $(0.97934 \AA, 0.97945 \AA$, and $0.97177 \AA$; peak, inflection point, remote) were collected on a Mar 300 CCD detector at SER-CAT beamline 22-ID, Argonne National Laboratory. Native data were collected at $\lambda=$ $1.0 \AA ̊$. All datasets were collected by using a single $1 \times 360^{\circ}$ pass to include Bijvoet pairs of reciprocal space for the P1 SeMet crystal and twofold redundancies in C2 for the native crystal, respectively.

Data Reduction and Scaling. Data were reduced, integrated, and scaled by using the HKL-2000 package (25), as reported in Table 3. Despite the similar crystal morphology of native and SeMet derivatives, they indexed in different space groups. Structure factor amplitudes were calculated by using TRUNCATE (26) and scaled to a reference dataset by using SCALEIT [27; except as noted, CCP4 (28) programs were used]. Statistical tests for the P1 selenomethionylated crystals (e.g., TRUNCATE, TWINNING) showed insignificant twinning.

Phasing and Refinement. We located the open Trp complex (1MB2) in the unit cell by molecular replacement [PHASER (29)] to obtain initial phases, with which we

1. Hammes-Schiffer S, Benkovic SJ (2006) Relating protein motion to catalysis. Annu Rev Biochem 75:519-541.

2. Bahar I, Chennubhotia C, Tobi D (2007) Intrinsic dynamics of enzymes in the unbound state and relation to allosteric regulation. Curr Opin Struct Biol 17:633-640.

3. Hu H, Yang W (2008) Free energies of chemical reactions in solution and in enzymes with ab initio quantum mechanics/molecular mechanics methods. Annu Rev Phys Chem 59:573-601.

4. Schiffer C, Hermans J (2003) Promise of advances in simulation methods for protein crystallography: Implicit solvent models. Time averaging refinement, and quantum mechanical modeling. Methods Enzymol 374:412-461.

5. Sharma S, Ding F, Dokholyan NV (2008) Probing protein aggregation using discrete molecular dynamics Frontiers Biosci 13:4795-4808.

6. Ding F, Tsao D, Nie H, Dokholyan NV (2008) Ab-initio folding of proteins with all-atom discrete molecular dynamics. Structure 16:1010-1018.

7. Franklin J, Koehl P, Doniach S, Delarue M (2007) MinActionPath: Maximum likelihood trajectory for large-scale structural transitions in a coarse-grained locally harmonic energy landscape. Nucleic Acids Res 35:W477-W482.

8. Kirby AJ, Younas M (1970) The reactivity of phosphate esters. Reactions of diesters with nucleophiles. J Chem Soc B 418:1165-1172.

9. Klinman J, Samuel D (1971) Oxygen-18 studies to determine the position of bond cleavage of acetyl-phosphate in the presence of divalent metal ions. Biochemistry 10:2126-2131.

10. Doublie S, Bricogne G, Gilmore C, Carter CW, Jr (1995) Tryptophanyl-tRNA synthetase crystal structure reveals an unexpected homology to tyrosyl-tRNA synthetase. Structure 3:17-31.

11. Retailleau P, et al. (2001) High-resolution experimental phases for tryptophanyl-tRNA synthetase (TrpRS) complexed with tryptophanyl-5'AMP. Acta Crystallogr 57:15951608.

12. Ilyin VA, et al. (2000) 2.9 A crystal structure of ligand-free tryptophanyl-tRNA synthetase: Domain movements fragment the adenine nucleotide binding site. Protein Sci 9:218-231.

13. Retailleau P, et al. (2003) Interconversion of ATP binding and conformational free energies by tryptophanyl-tRNA synthetase: Structures of ATP bound to open and closed, pre-transition conformations. J Mol Biol 325:39-63.

14. Kapustina M, Weinreb V, Li L, Kuhlman B, Carter CW, Jr (2007) A conformational transition state accompanies amino acid activation by $B$. stearothermphilus tryptophanyl-tRNA synthetase. Structure 15:1272-1284.

15. Retailleau P, Weinreb V, Hu M, Carter CW, Jr (2007) Crystal structure of tryptophanyltRNA synthetase complexed with adenosine-5' tetraphosphate: Evidence for dissociative character in the transition state for amino acid activation by class I aminoacyl-tRNA synthetases. J Mol Biol 369:108-128. located 166 of the 180 Se sites in anomalous difference Fourier maps. Parameter refinement in SHARP (30) allowed location of the remaining Se atoms. Each wavelength was run as a separate SAD experiment when we determined that restraining $X Y Z, O C C$, and $B$ to be identical for all 3 wavelengths was not useful, perhaps because each dataset was collected after translating the crystal. Experimental phases were improved by solvent flipping (SOLOMON) and NCS averaging. Coordinate refinement [BUSTER 2.0 (31)] was interspersed with intensive manual model rebuilding [COOT (32)]. Imposing idiosyncratic noncrystallographic symmetry equivalences between small and Rossmann fold domains afforded improvement in both electron density and the crystallographic $R_{\text {free }}$.

MAP Trajectory Generation. A molecular transition path between the 2 forms of TrpRS was calculated by the MinActionPath method described in ref. 7. The program can now handle up to 10,000 atoms (M.D. and P.K., unpublished data). The parameters of the computation are: cutoff distance set to $8.0 \AA$; elastic constants, $C$, were set to $0.02 \mathrm{kcal} \cdot \mathrm{mol}^{-1} \cdot \AA^{-2}$ for both states with $\Delta G=0$ between states. All common atoms of the 2 forms were used, including ATP, $\mathrm{Mg}^{2+}$, and Trp. The influence of (i) $\Delta G=0$ or $-1.8 \mathrm{kcal} / \mathrm{mol}$ and (ii) $C=0.02$ or $0.2 \mathrm{kcal} \cdot \mathrm{mol}^{-1} \cdot \AA^{-2}$ were found to give qualitatively the same results.

ACKNOWLEDGMENTS. We thank the staff at the South East Region Collaborative Access Team beamline 22ID; G. Bricogne for substantive discussions about phase determination and refinement and J. Hermans for referring us to Berendsen's work on statistical errors in time series; Marshall Edgell and Jan Hermans for valuable input to the preparation and interpretation of Figs. 1 and 4; and Laurie Betts for assistance with X-ray data collection. This work was supported by National Institutes of Health Grants GM 48519 and 78227. P.L. is supported by a Postdoctoral Fellowship Grant from the Thailand Center of Excellence for Life Sciences and the University of North Carolina School of Medicine.

16. Fersht A (1988) Dissection of the structure and activity of an enzyme. Design of Enzymes and Enzyme Models, ed Kaiser ET (Robert A. Welch Foundation, Houston, TX), Vol 31, pp 159-182.

17. Fersht AR (1987) Dissection of the structure and activity of the tyrosyl-tRNA synthetase by site-directed mutagenesis. Biochemistry 26:8031-8037.

18. Kapustina M, Carter CW, Jr (2006) Computational studies of tryptophanyl-tRNA synthetase: Activation of ATP by induced-fit. J Mol Biol 362:1159-1180.

19. Carter CW, Jr (2005) Tryptophanyl-tRNA synthetases. The Aminoacyl-tRNA Synthetases, eds Ibba M, Francklyn C, Cusack S. (Landes Biosciences/Eurekah.com, Georgetown, TX), pp 99-110.

20. Miller BG, Snider MJ, Short SA, Wolfenden R (2000) Contribution of enzymephosphoribosyl contacts to catalysis by orotidine $5^{\prime}$-phosphate decarboxylase. Biochemistry 39:8113-8117.

21. Carlow DC, Short SA, Wolfenden R (1998) Complementary truncations of a hydrogen bond to ribose involved in transition state stabilization by cytidine deaminase. Biochemistry 37:1199-1203.

22. Roach JM, Sharma S, Kapustina M, Carter CW, Jr (2005) Structure alignment via Delaunay tetrahedralization. Proteins Struct Funct Bioinform 60:66-81.

23. Straatsma TP, Berendsen HJC, Stam AJ (1986) Estimation of statistical errors in molecular simulation calculations. Mol Phys 57:89-95.

24. Carter CW, Jr, Yin Y (1994) Quantitative analysis in the characterization and optimization of protein crystal growth. Acta Crystallogr D 50:572-590.

25. Otwinoski Z, Minor W (1997) Processing of x-ray diffraction data collected in oscillation mode. Methods Enzymol 276:307-326.

26. French GS, Wilson KS (1978) On the treatment of negative intensity observations. Acta Crystallogr A 34:517-525.

27. Howell L, Smith D (1992) Identification of heavy-atom derivatives by normal probability methods. J App/ Crystallogr 25:81-86.

28. Collaborative Computing Project No 4 (CCP4) (1991) The SRC(UK) Collaborative Computing Project No 4: A Suite of Programs for Protein Crystallography (Daresbury Laboratory, Daresbury, U.K.).

29. McCoy AJ, et al. (2007) Phaser crystallographic software. J App/ Crystallogr 40:658674.

30. de La Fortelle E, Bricogne G (1997) Maximum-likelihood heavy-atom parameter refinement for multiple isomorphous replacement and multiwavelength anomalous diffraction methods. Methods Enzymol 276:472-494.

31. Blanc E, et al. (2004) Refinement of severely incomplete structures with maximum likelihood in BUSTER-TNT. Acta Crystallogr D 60:2210-2221.

32. Emsley P, Cowtan K (2004) Coot: Model-building tools for molecular graphics Acta Crystallogr D 60:2126-2132. 